\title{
"Droplet Interaction Technologies" (DROPIT): Selected Results of the International Research Training Group GRK 2160
}

\author{
B. Weigand ${ }^{* 1}$, G. Lamanna ${ }^{1}$, S. Tonini ${ }^{2}$, G.E. Cossali² \\ ${ }^{1}$ Institute of Aerospace Thermodynamics, University of Stuttgart, Germany \\ ${ }^{2}$ Dep. of Engineering and Applied Science, University of Bergamo, Italy \\ *Corresponding author: bernhard.weigand@itlr.uni-stuttgart.de
}

\begin{abstract}
The International Research Training Group GRK 2160 (DROPIT), active since Oct. 2016, focuses on droplet interaction technologies, which have a large number of applications in a variety of technological processes. The main objective of DROPIT is to identify and to investigate the mechanisms through which small-scale interactions at the interface couple with and influence large scale features in the main flow. DROPIT is a joint initiative of the University of Stuttgart in Germany, the University of Bergamo and the University of Trento in Italy. The project consists of 17 subprojects, which are structured into three main research areas, involving researchers from a large number of different disciplines. The project consists of an extensive qualification program which aims at fostering the education of young scientists and providing them the knowledge and skills to conduct independent research. This paper provides an overview of the structure and the research activities within DROPIT as well as on the qualification program implemented. Selected research results are shown and discussed. The main purpose of this paper is to familiarize colleagues with this extensive research effort in the area of droplet interaction technologies and to exchange ideas and promote future collaboration with others in this field.
\end{abstract}

\section{Keywords}

Droplet interaction, numerical methods, experimental methods, theoretical methods

\section{Introduction}

Droplet interaction technologies find application in a large number of technological and industrial processes. These include spray cooling, spray drying absorption, droplet collisions for the generation of powders and encapsulated material, drop evaporation and droplet-wall interaction in internal combustion engines. In all these applications, small scale fluid dynamics may have a huge impact on large scale flow pattern, leading to drag reduction, heat transfer enhancement or depression, phase transition kinetics (e.g. drop condensation or nucleate boiling), acoustic impedance and optical reflection. The consequences of the presence of different length scales on macroscopic properties and the associated amplification of surface transport is still largely unexplored, mostly limited to the formulation of empirical correlations based solely on macroscopic observations. Here, it is the aim of the GRK 2160 to provide a systematic study of the interdependencies between small-scale and large-scale dynamics in the field of droplet interaction technologies. Due to the complexity of the problem, the analysis of such micro/macro interactions is not limited to one single aspect. Rather, an integrated approach is chosen that evolves along three parallel pathways, namely a numerical, experimental and theoretical approach [21, 22]. More detailed information concerning the International Research Training Group GRK 2160 can be found online: www.project.unistuttgart.de/dropit/. 


\section{Structure of the International Research Training Group GRK2160/1 (DROPIT)}

The International Research Training Group is structured into three main research areas (RAA to RA-C), comprising 17 subprojects, which are listed below together with the responsible principle investigators:

\section{Research Area A: Drop-Gas Interaction}

SP-A1: Modelling of deformed, multi-component liquid drop evap. (Cossali, Tonini, Weigand)

SP-A2: Multi-scale modelling of the evaporation process (Munz, Rohde, Dumbser)

SP-A3: Gas-kinetic simulation of micro droplet - gas interaction (Fasoulas, Lamanna, Bassi)

SP-A4: Numerical methods for compressible multiphase flows with complex equations of state (Dumbser, Munz)

SP-A5: Modelling of spray evaporation (Cossali, Tonini)

SP-A6: Mathematical and numerical modelling of droplet dynamics in weakly compressible multi-component flows (Beck, Bassi)

\section{Research Area B: Drop-Wall Interaction}

SP-B1: Droplet collisions with solid superhydrophilic surfaces (Roth, Weigand, Cossali)

SP-B2: Drop impact/deposition onto micro-structured hydrophobic and superhydrophobic surfaces (Santini, Roth)

SP-B3: Characterization of porous media by X-ray micro computed tomography (Santini, Ertl)

SP-B4: Compressible effects in droplet interactions with textured walls (Rohde, Bassi)

SP-B5: Numerical computation for drop impact on textured surfaces (Weigand, Dumbser)

SP-B6: Upscaling of coupled free-flow porous media flow processes (Helmig, Santini)

\section{Research Area C: Drop-Liquid Interaction}

SP-C1: Micro and macro drop impact dynamics with miscible liquids (Lamanna, Tonini)

SP-C2: Single and multiple drop impact into a deep pool (Santini, Cossali, Helmig)

SP-C3: High-order num. methods for multi-component incomp. flows in pools (Bassi, Munz)

SP-C4: Visualisation of droplet-liquid interaction (Ertl, Santini)

SP-C5: Dev. of novel optical techniques for micro-fluid dyn. (Weigand, Lamanna, Cossali)

In RA-A, the research activities focus mainly on the optimization of evaporation models for sprays. The objective is to improve the accuracy of current models by including effects of small-scale processes on the global evaporation rate. This entails non-equilibrium, thermodynamic effects at the drop interface (SP-A3), local curvature effects (SP-A1, SP-A5) and multi-component effects (SP-A1, SP-A6). In addition, subprojects (SP-A2, SP-A4, SP-A6) focus on the development of numerical models for droplet dynamics and evaporation by taking into account multi-scale and/or compressibility effects.

In RA-B, the research activities are mainly dedicated to the understanding and modelling of drop impact on solid structured surfaces. This includes textured surfaces (SP-B4, SP-B5), pretreated surfaces with hydrophobic or hydrophilic coatings (SP-B1, SP-B2) and porous structures (SP-B3, SP-B6). The objective is to gain detailed understanding on how micro-scale structures can affect macroscopic detectable quantities and transport processes.

In RA-C, the applications are focused on drop collisions (onto a film or into a pool) with miscible fluids. In SP-C1 and SP-C5, the main objective is to understand how miscibility-gaps and thinfilms micro-dynamics influence the large-scale impact dynamics and resulting drop size distribution. Deep pool dynamics for drop impact is investigated experimentally and numerically in SP-C2 and SP-C3, respectively. Visualisation methods support a better understanding of the results and are developed in SP-C4. 


\section{Qualification program}

The increased interdependence of economies and technologies together with the need of reducing the ecological footprint of industrial processes requires excellently qualified researchers and engineers with an interdisciplinary background and international experience. Our qualification program is especially designed to meet these requirements. Interdisciplinary elements are strengthened to enhance the quality of the education. Here, special emphasis is placed on basic knowledge in the natural sciences and in engineering as well as in mathematics, always applied to the topic at hand. The experiences of former Research Training Groups are incorporated in the present study program, enriched by some new elements to attract excellent students as well as to take into consideration the growing importance of experiences abroad. Special emphasis is set on the following elements:

- An individual study program comprising graduate-level lectures,

- a yearly joint doctoral meeting to discuss the scientific progress of the IRTG with the participation of members from the advisory board and all involved PIs,

- lecture series of scientific members of DROPIT and guest researcher,

- seminars of external scientists,

- annual organization of an international workshop,

- a three-yearly international summer school, organized directly by the doctoral researchers to foster their self-management skill and

- an individually conceived stay abroad for six months.

The home institutions of the scientific members of DROPIT are the University of Stuttgart, the University of Bergamo and the University of Trento. All doctoral researchers participating in the International Research Training Group (IRTG) GRK 2160 are associated to one of the available graduate academies: GRADUS (Stuttgart), ISA (Bergamo) or DICAM (Trento). Due to the regional separation, modern web-based communication techniques are routinely used. Research data of the subprojects is exchanged using file transfer servers.

\section{Results and Discussion}

In the following some current results from selected subprojects are shown.

\section{SP-A4 (S. Chiocchetti, M. Dumbser, C.-D. Munz)}

Recently, several first order reductions of systems of higher order partial differential equations have been forwarded. Some examples include hyperbolic reformulations of the Serre-GreenNaghdi equations [1], a unified first order hyperbolic description of fluid and solid mechanics [2], as well as a first order reduction of the Z4 formulation of the Einstein field equations [3]. Such an interest is motivated by the verifiability of the well-posedness of initial-value problems involving this type of equations, as well as by the implementability of the models in the context of general-purpose Finite Volume and Discontinuous Galerkin Finite Element solution codes requiring a first order form of the governing equations. We are concerned here with presenting our results in regards to the development of a mathematical model for two-phase flow with surface tension and its solution using high order Discontinuous Galerkin schemes of the ADER family [4]. The model is a modification of the one originally proposed in [5], which replaces a diffuse representation of interfaces of the Volume-of-Fluid type (by means of a scalar tracer), with one using a vector variable indicating the localisation and orientation of interfaces separating two fluids. This so-called interface field is initially computed as the gradient of a classical VoF-type passive tracer or colour function, but its components are subsequently evolved as independent variables. For example, in 2D, a circular droplet may be represented by the presence of a circular region of high concentration, tapering out to an environment in 
which the tracer is absent, which would translate to a vector field with stronger intensity in a ring at the interface (where the gradient of the scalar would be strongest). An interesting aspect of this description of interfaces is that it clearly localizes in space the presence of an energy field associated with surface forces, obtained by multiplying the Euclidean norm of the interface field vector by the constant surface tension coefficient. A recent result of our investigations [8] is that, in its original weakly hyperbolic formulation [5], the model is not suitable for discretisation with explicit high order methods, unless specific ad-hoc strategies are adopted to stabilise the computations. Therefore, our interest has been devoted to constructing a strongly hyperbolic variant of the equations and we found that either a symmetrisation strategy based on a non-conservative form of the governing equations (like in [6]) or hyperbolic GLM cleaning of curl involution constraints of the model (see [7]) can be employed in order to recover a strongly hyperbolic formulation.

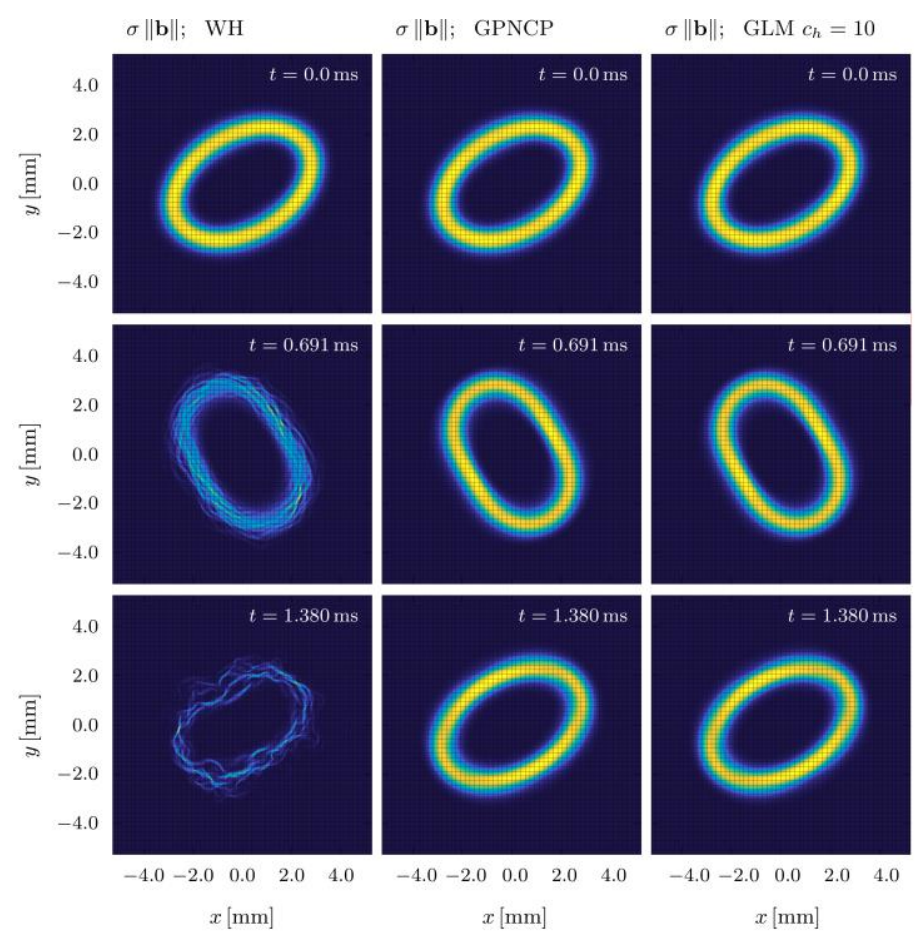

Figure 1. Oscillation of an elliptical droplet, computed with a sixth order ADER Discontinuous Galerkin scheme: the first column shows the behavior of the weakly hyperbolic model, which quickly develops filament-like instabilities and grows unstable; the second column depicts the behavior of the non-conservative symmetrised variant of the model (GPNCP); the third column is obtained from the GLM curl cleaning augmented equations.

The symmetrising approach consists in modifying the momentum and energy equations by adding suitable multiples of the curl constraint. This leads to a loss of conservativity of these equations, but direct computation of the eigenstructure of the resulting model yields a full set of linearly independent eigenvectors, and numerical investigations clearly show the advantages with respect to the weakly hyperbolic model, namely that computations are stable and compare accurately to exact analytical predictions. The hyperbolic GLM curl cleaning requires that one solves an augmented system of governing equations, obtained by introducing a new vector quantity, dual to the interface field, that propagates curl errors away from where they have been generated, avoiding local accumulation of curl violations, as well as yielding a strongly hyperbolic modified system that also retains conservation of momentum and energy. As a numerical example, we report a comparison of the models in capturing the global dynamics of an elliptical droplet, which oscillates under the effect of surface tension due to being initially set up out of equilibrium. In Fig. 1 one can see that, using the weakly hyperbolic model $(\mathrm{WH})$, the initially elliptical interface quickly deteriorates into spurious 
filament structures leading to an early stop of the computations. Meanwhile, the two proposed strongly hyperbolic variants of the governing equations (GPNCP and GLM) allow a stable and accurate simulation of the droplet dynamics to be carried out.

\section{SP-A6 (J. Zeifang, A. Beck, F. Bassi)}

While most numerical frameworks are either designed for the simulation of compressible or incompressible flows, the aim of this project is to develop numerical methods which are capable to handle flows ranging from weakly compressible regimes to compressible regimes. Such regimes frequently occur simulating droplet dynamics, as the speed of sound of liquids is often much larger than for gases. The challenges developing such methods stem from the change of characteristics of the underlying equation system. While the compressible Euler equations have a hyperbolic character and the density is strongly coupled with the thermodynamic pressure, the incompressible Euler equations have a hyperbolic-elliptic character. Additionally, the pressure is no longer coupled to the density but serves as a hydrodynamic pressure being linked to the velocity. In between the incompressible and compressible regime lies the weakly compressible region, where the propagation speeds of the different waves differ by orders of magnitude. This poses difficulties for the design of numerical algorithms. The high order code FLEXI of the Institute of Aerodynamics and Gas Dynamics at the University of Stuttgart has been shown to be well suited for the application to compressible flows. It uses the discontinuous Galerkin spectral element method (DGSEM) for the spatial and explicit Runge-Kutta schemes for the temporal discretization [9]. In the scope of the current project, FLEXI has been extended to a mixed implicit-explicit (IMEX) time discretization with IMEX Runge-Kutta schemes to account for the different propagation velocities, which are present in weakly compressible flows. The use of the IMEX time discretization enables the use of flux splitting techniques. The idea of flux splitting is to separate fast waves, associated with acoustics, and slow convective waves.
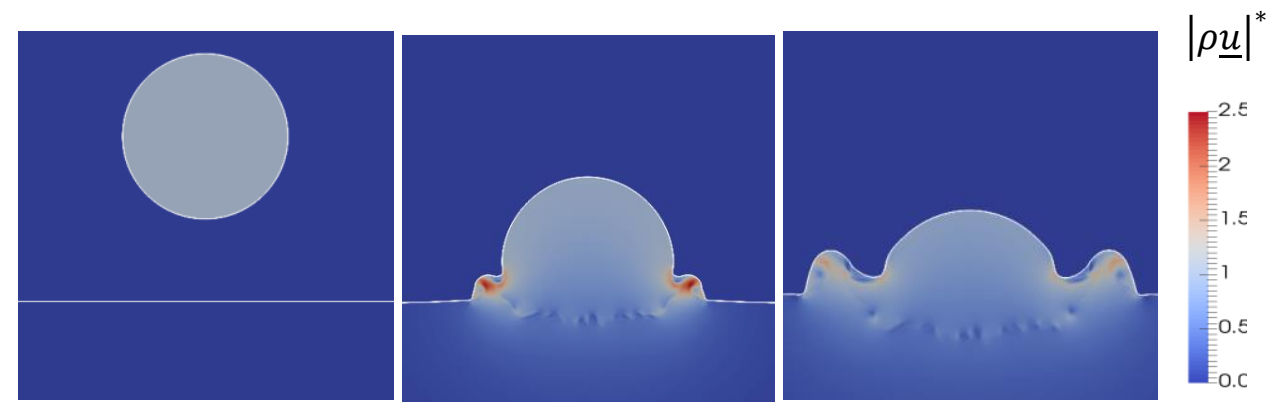

Figure 2. Temporal evolution of relative momentum of impinging drop at $\tau=0$ (left), $\tau=0.75 \tau^{*}$ (middle) and $\tau=1.0 \tau^{*}$ (right). The phase boundary is indicated with a white line.

While the fast waves are treated implicitly, the convective waves are treated explicitly in time. This ensures an accurate discretization of the phenomena of interest. In that sense, the RSIMEX splitting from Kaiser and Schütz [10] for the compressible isentropic Euler equations has been applied to the DGSEM. Its favourable properties, with respect to accuracy and efficiency have been shown in [11] and the extension to the full Euler equations in [12]. In the scope of this project, IMEX time discretization and flux splitting have been applied to a sharp interface level-set ghost fluid method, based on the work of Fechter and Munz [13]. The example in Fig. 2 shows a water drop impact into a pool, surrounded by air. It has been calculated with a fourth order method in space and time in the bulk phases and with approximately 140 degrees of freedom per drop diameter. An implicit time discretization for the fluid and an explicit time discretization for the level-set transport have been used, which allows to use a time step that 
is approximately 10 times larger than for a comparable fully explicit scheme. The setup is characterized by the impact Weber number $W e=\rho_{l} u_{0}^{2} D / \sigma=23$ and the Mach number $M a=$ $u_{0} / c_{g}=0.115$. Thereby, $\rho_{l}$ denotes the density of the liquid, $u_{0}$ the impact velocity, $D$ the droplet diameter, $\sigma$ the surface tension coefficient and $c_{g}$ the speed of sound of the gas. The ratios of liquid and gaseous densities and speed of sounds are given by $\rho_{l} / \rho_{g}=862.6$ and $c_{l} / c_{g}=4.4$. Note that the influence of the viscosity and the gravity is neglected, and the drop centre is initialized $1.0 D$ above the pool surface with depth $4 D$. The characteristic time scale $\tau^{*}=D / u_{0}$ relates the droplet size to the impact velocity and the relative momentum $|\rho \underline{u}|^{*}=$ $|\rho \underline{u}| /\left|\rho_{l} \underline{u}_{0}\right|$ relates the current momentum to the initial momentum of the droplet.

\section{SP-B4 \& SP-C3 (L. Ostrowski, F. Massa, C. Rohde, F. Bassi)}

In this joint work of the subprojects B4 and C3 the aim is to quantify compressibility effects on the liquid component during drop impact scenarios. On one hand, in SP-B4 a compressible Navier-Stokes-Allen-Cahn (NSAC) phase field model is used to simulate droplet impacts, where the liquid and the gaseous phase are both modelled as compressible fluids [14]. On the other hand, in SP-C3 a variable-density incompressible (VDI) model is considered to simulate the interaction between incompressible multi-component fluids [15]. An intermediate perspective between the two subprojects has been recently presented in [16]. In particular, a novel Incomp.-Compr. Navier-Stokes-Cahn-Hilliard (ICNSCH) phase field model has been introduced, which takes compressibility of the gaseous phase into account, whereas the liquid phase is assumed to be incompressible. The comparison of these different models enables the possibility to investigate the importance of the compressibility of the liquid phase, when simulating droplet impact scenarios. The ICNSCH phase field model is derived on basis of the quasi-incompressible Navier-Stokes-Cahn-Hilliard model of Lowengrub and Truskinovsky (LTNSCH) [17], where both phases are incompressible. As a first step, we analysed this model with respect to the low-Mach number limit and surface tension. Using an asymptotic low Mach number analysis, we formally showed that in a particular asymptotic regime the ICNSCH model attains the LTNSCH model. The surface tension contribution, included implicitly in the model through the energy-based derivation, has common features with both the LTNSCH and the compressible NSAC models. In [16] a numerical method to discretise the ICNSCH phase field model has been proposed but not implemented. It is a special instance of the Discontinuous Galerkin (DG) methods, the so-called energy consistent DG schemes. As peculiar feature this method fulfills the mechanical energy inequality at the discrete level, thus preventing the formation of any parasitic currents. However, it is restricted to second order accuracy with respect to the time discretization. Due to this drawback, in this joint work we aim to design a novel solver based on a DG method which can achieve higher order accuracy, both in space and time. The idea is to use the numerical framework available from the code MIGALE used in SP-C3. This code relies on a DG method for the space discretization and on high-order Rosenbrock and ESDIRK implicit schemes for the time integration. The discontinuous nature of the numerical solution at the inter-element boundaries is handled by introducing suitable inviscid and viscous numerical fluxes. For the former flux a Godunov flux based on the exact solution of a local Riemann problem is typically employed. For the latter flux the BR2 scheme [18] is used.

When applying the DG method to the ICNSCH model, specific care must be devoted to the treatment of: (i) the numerical Godunov flux, (ii) the surface tension contribution and (iii) the chemical potential. Preliminary steps have already been done for points (i) and (ii). For what concerns the Godunov flux, we take inspiration from the work of Bassi et al. [19] and define 
an artificial Equation of State (EoS) for the liquid phase [20]. In such a way the hyperbolicity of the local Riemann problem is recovered and an exact solution and thus a numerical flux can be defined. The surface tension contribution, instead, is formulated as the divergence of the capillarity tensor. Since this tensor is defined by means of the gradients of the phase field variable, the idea is to apply the BR2 scheme.
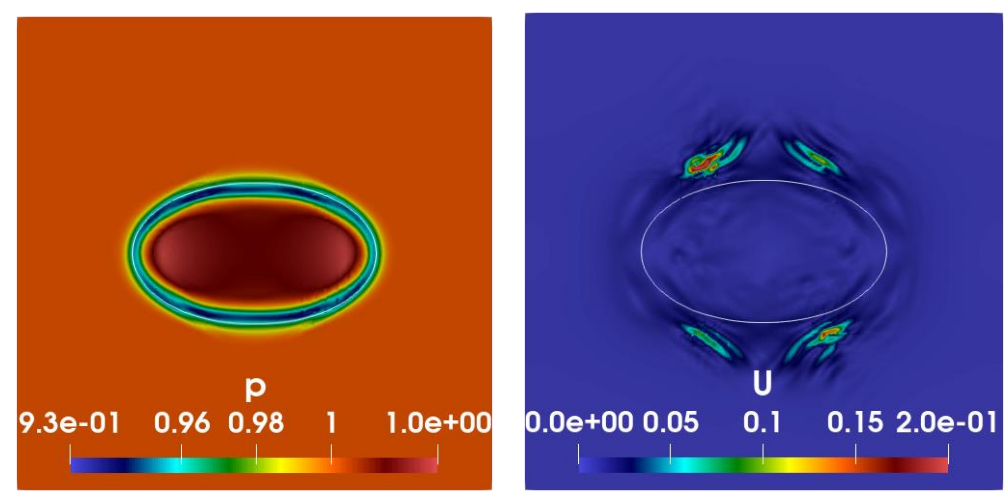

Figure 3. Pressure $(p)$ and absolute value of velocity $(U)$ contours after one period of the oscillation (inviscid oscillating droplet test case with air and water). The white line is the isoline of the average density between the two components.

As a test of the proposed artificial EoS approach, the new Riemann solver has been implemented in the incompressible single-phase code MIGALE. Excellent results have been achieved in terms of space as well as time convergence rates. Moreover, preliminary results on the implicit Large Eddy Simulation of a turbulent flow over periodic hills are showing good agreement with experimental and numerical references. As future work the application of the artificial EoS approach to the ICNSCH model will be investigated. Moreover, as a test of the implementation of the surface tension contribution, an equivalent formulation for the VDI flow model has been implemented and tested with success for the oscillating droplet test case. Figure 3 shows the pressure field and the velocity magnitude field obtained after one period of the oscillation.

\section{Summary and Conclusions}

This paper shows the structure, the qualification program and some current, selected results of the International Research Training Group GRK 2160: "Droplet Interaction Technologies". This research project, which is running since October 2016, is a joint initiative of the University of Stuttgart in Germany, the University of Bergamo and the University of Trento in Italy. The main goal of the project is to investigate changes in macroscopic flow properties due to the coupling between small scale effects with large scale effects on flow dynamics. Selected results, which have been reported in this paper, demonstrate the recent progress, which has been achieved in the project.

\section{Acknowledgments}

The members of the GRK 2160 would like to thank the Deutsche Forschungsgemeinschaft (DFG) for the financial support of the International Research Training Group GRK 2160 under the project number 270852890.

\section{Nomenclature}

C speed of sound [ $\left.\mathrm{m} \mathrm{s}^{-1}\right]$

$D \quad$ droplet diameter [m]

$\mathrm{Ma}$ Mach number [-] 


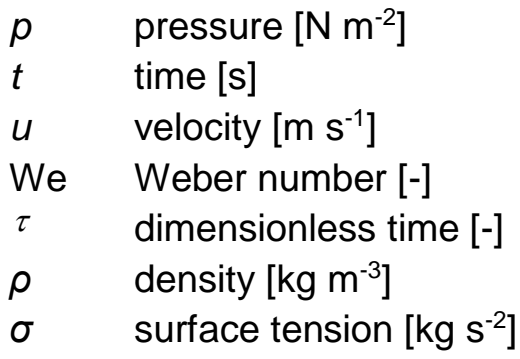

\section{References}

[1] Favrie, N. and Gavrilyuk, S., 2017, Nonlinearity 30(7), pp.2718-2736.

[2] Peshkov I. and Romenski, E., 2016, 28, pp.85-104.

[3] Dumbser, M., Guercilena, F., Köppel, S., Rezzolla, L.and Zanotti, O., 2018, Physical Review D 97:084053.

[4] Dumbser, M., Balsara, D.S., Toro, E.F.and Munz, C.-D., 2008, Journal of Comp. Physics, 227(18), pp. 8209-8253.

[5] Schmidmayer, K., Petitpas, F., Daniel, E. Favrie, N. and Gavrilyuk, S., 2017, Journal of Comp. Physics, 334(1), pp.468-496.

[6] Powell, K.G., Roe, P.L., Linde, T.J., Gombosi, T.I. and De Zeeuw, D.L., 1999, Journal of Comp. Physics, 154(2), pp. 284-309.

[7] Dedner, A., Kemm, F., Kröner, D., Munz, C.-D., Schnitzer, T. and Wesenberg, M.2002, Journal of Computational Physics, 175, pp. 645-673.

[8] Chiocchetti, S., Peshkov, I., Gavrilyuk, S. and Dumbser, M., 2021, Journal of Comp. Physics, 426, 109898.

[9] Hindenlang, F., Gassner, G., Altmann, C., Beck, A., Staudenmaier, M. and Munz, C.-D., 2012, Computers and Fluids, 61, pp. 86-93.

[10] Kaiser, K.and Schütz, J., 2017, Comm. in Computational Physics, 22 (4), pp. 1150-1174.

[11] Zeifang, J., Kaiser, K., Beck, A., Schütz, J. and Munz, C.-D., 2018, Comm. in Applied Mathematics and Comp. Science, 13 (2), pp. 243-270.

[12] Zeifang, J., Schütz, J., Kaiser, K. and Beck, A., Lukácová-Medvid'ová, M., Noelle, S., 2020, Comm. in Comp. Physics, 27 (1), pp. 292-320.

[13] Fechter, S. and Munz, C.-D., 2015, Int. J. for Num. Meth. in Fluids, 78 (7), pp. 413-435.

[14] Ostrowski, L., Massa, F. and Rohde, C: A, 2020, Droplet Int. and Spray Proc., Springer.

[15] Massa, F., Bassi, F., Botti, L. and Colombo, A., 2020, Droplet Interaction and Spray Processes, Springer, Heidelberg, Berlin.

[16] Repossi, E., Rosso, R. and Verani, M., 2017, Calcolo 54, pp. 1339-1377.

[17] Lowengrub, J. and Truskinovsky, L., 454. Proc. Royal Soc. Lond. A., 1998.

[18] Bassi, F., Rebay, S., Mariotti, G., Pedinotti, S. and Savini, M., 1997, Tech. Instit. Belg., pp. 99-108.

[19] Bassi, F., Crivellini, A., Di Pietro, D.A. and Rebay, S., 2006, J. Comp. Phys. 218 (2), pp 794-815.

[20] Ostrowski, L. and Massa, F., 2019, Proc. of DIPSI Workshop 2019, Bergamo, Italy, 2019.

[21] Lamanna, G. Tonini, S. Weigand, B. and Cossali. G.E., July 22.-26. 2018, 14th Triennial International Conference on Liquid Atomization and Spray Systems.

[22] Cossali, G.E., Weigand, B., Lamanna, G. and Tonini, S., Sept. 2-4 2019, $29^{\text {th }}$ Conf. on Liquid Atomization and Spray Systems. 